\title{
Exploring Travel Time Distribution and Variability Patterns Using Probe Vehicle Data: Case Study in Beijing
}

\author{
Peng Chen $\mathbb{D}^{1,},{ }^{1,2}$ Rui Tong, ${ }^{1}$ Guangquan Lu $\left.\mathbb{D}\right)^{1,2}$ and Yunpeng Wang ${ }^{1,2}$ \\ ${ }^{1}$ Beijing Key Laboratory for Cooperative Vehicle Infrastructure Systems and Safety Control, School of Transportation Science and \\ Engineering, Beihang University, Xue Yuan Road No. 37, Hai Dian District, Beijing 100191, China \\ ${ }^{2}$ Beijing Advanced Innovation Center for Big Data and Brain Computing, Beihang University, \\ Xue Yuan Road No. 37, Hai Dian District, Beijing 100191, China \\ Correspondence should be addressed to Peng Chen; cpeng@buaa.edu.cn
}

Received 6 November 2017; Accepted 27 March 2018; Published 7 May 2018

Academic Editor: Yair Wiseman

Copyright (C) 2018 Peng Chen et al. This is an open access article distributed under the Creative Commons Attribution License, which permits unrestricted use, distribution, and reproduction in any medium, provided the original work is properly cited.

Exploring travel time distribution and variability patterns is essential for reliable route choices and sophisticated traffic management and control. State-of-the-art studies tend to treat different types of roads equally, which fails to provide more detailed analysis of travel time characteristics for each specific road type. In this study, based on a vast amount of probe vehicle data, 200 links inside the Third Ring Road of Beijing, China, were investigated. Four types of roads were covered including urban expressways, auxiliary roads of urban expressways, major roads, and secondary roads. The day-of-week distributions of unit distance travel time were first analyzed. Kolmogorov-Smirnov test, Anderson-Darling test, and chi-squared test were employed to test the goodness-of-fit of different distributions and the results showed lognormal distribution was best-fitted for different time periods and road types compared with normal, gamma, and Weibull distribution. In addition, four reliability measures, that is, unit distance travel time, coefficient of variation, buffer time index, and punctuality rate, were used to explore the day-of-week travel time variability patterns. The results indicated that urban expressways, auxiliary roads of urban expressways, and major roads have regular and distinct morning and afternoon peaks on weekdays. It is noteworthy that in daytime the travel times on auxiliary roads of urban expressways and major roads share similar variability patterns and appear relatively stable and reliable, while urban expressways have most reliable travel times at night. The results of analysis help enable a better understanding of the volatile travel time characteristics of each road type in urban network.

\section{Introduction}

Nowadays, high traffic demand and limited road capacities make people spend much more time on their daily journeys. Travel time reliability (TTR), defined as the level of consistency of travel conditions over time [1], has been increasingly considered as an important measure of the performance of transportation systems as well as travelers' perceptions of such performance. In order to comprehensively characterize TTR, travel time distribution (TTD) and variability patterns need to be explored as a prior, which is essential for reliable route choices and sophisticated traffic management and control [2].

Thanks to advanced traffic sensing technologies, various travel time related information can be collected conveniently nowadays. The technologies essentially include station-based traffic state measurement (e.g., loop detector, video camera, and microwave sensor) and point to point travel time collection (e.g., automatic vehicle identification systems, license plate recognition systems, mobile, Bluetooth, and probe vehicles). The acquisition results of station-based devices strongly depend on the spatial layout and fixed position of traffic detectors. In contrast, probe vehicles equipped with the global positioning system (GPS) could travel all over the network and record the travel time and location information of vehicles at a certain interval. These data are known as probe vehicles data, representing the relatively complete operation conditions for urban traffic. With increasing amounts of data available, there has been a surge of literature devoted to the analysis of TTR and TTD in recent years. 
Urban traffic times are essentially volatile due to various influencing factors, for example, weather, road types, roadway geometry, traffic control, accident, and varying traffic demands. Martchouk et al. [3] compared the travel time data collected using Bluetooth detection during good and bad weather on freeway segments, and found that there was an increase in travel time that continues throughout the snowstorm event. A number of studies considered the possible impact of roadway geometry on the travel time of urban arterials, such as number of lanes and left turn lane types $[4,5]$. Chen et al. [6] investigated travel time data on one urban arterial incorporating the effects of traffic signal timing, and the results demonstrated that traffic control has a distinct effect on urban travel time. Several studies analyzed the relationships between travel time or speed and volume-tocapacity ratio of expressways $[3,7,8]$ and found that the travel time variability is greatly affected by volume-to-capacity ratio.

It is noteworthy that most studies on travel time analysis employed travel time data from only one road type $[3,9,10]$ or treated different types of roads equally $[5,11]$, which failed to provide more detailed analysis of travel time characteristics for each specific road type. In practice, travel times of different road types are supposed to have diverse variability patterns due to different road characteristics and capacities and attractiveness for travelers. Only limited study [12] adopted the travel time data from highway and urban roads to identify the relatively coarse distinction caused by two facility types. TTR needs to be investigated at fine grained levels of spatial and temporal aggregation. Thus, based on a vast amount of probe vehicle data, this study aims to explore the TTD and variability patterns for different road types (e.g., urban expressways, auxiliary roads of urban expressways, major roads, and secondary roads) in typical time periods (e.g., peak versus off-peak, weekday versus weekend).

The remainder of this paper is organized as follows. Section 2 provides a literature review of TTD and variability patterns, followed by a detailed description of the datasets used for analysis. In Section 3, we investigate and compare the goodness-of-fit of four typical distributions to travel times for different road types in different time periods. Then, travel time variability (TTV) patterns are identified and discussed in terms of four reliability measures in Section 4. Last, summary and conclusions are given in Section 5.

\section{Literature Review}

2.1. Travel Time Distribution. Most existing studies on TTV put significant effort into identifying the best statistical model for fitting travel times. The fitted distributions can be categorized into three main classes, that is, single-mode distributions [9, 13-16], multimode distributions [6, 17-19], and truncated distributions [4, 20, 21].

Single-mode distributions apply one kind of standard distributions to characterize travel time, like normal, lognormal, gamma, Weibull, burr, and other distributions that are commonly used. For example, Emam and Al-Deek [15] tested the lognormal, gamma, Weibull, and exponential distributions for representing travel time data of a freeway from weekdays and found that a lognormal distribution was bestfitted. Nie et al. [22] adopted gamma distribution to model travel time on arterial and local streets during morning peak, mid-of-day, and evening peak. Lei et al. [9] compared Generalized extreme, Weibull, burr, normal, gamma, lognormal, Generalized Pareto, and other distributions for modeling travel times on urban expressways with varying levels of service and concluded that generalized extreme and Generalized Pareto are preferable fits than others. Kieu et al. [16] selected burr, gamma, lognormal, normal, and Weibull to model public transport travel time and found that lognormal can be recommended as the descriptor because of its desirable performance and attractive mathematical characteristics. Single-mode distribution is simple and the parameter(s) of the distribution is easy to estimate. Such a fitting approach is usually adopted by traffic engineers with its attractive mathematical characteristics, though the goodness-of-fit may be not quite desirable due to substantially different free flow and congested conditions.

Multimode distribution appears more accurate because more than one component will be used to model travel time states. The components can come from one or multiple standard distributions. For example, Chen et al. [6] compared different regression mixture models including the model whose all components are Gaussian distributions and the model with mixture of Gaussian and lognormal distributions to estimate urban arterial travel time. Guo et al. [18] discussed multistate versions of normal, gamma, and lognormal distributions, and the results showed that the multistate lognormal model consistently outperforms other models, especially during peak hours. Benefiting from its multistate nature, multimode distribution is capable of adapting the different characteristics of travel time states in urban roads, for example, caused by the diversity of vehicle model (e.g., cars versus buses) and signal timings (e.g., green light versus red light). Nevertheless, the proportion and parameter(s) of each component are usually determined by extra estimation methods, such as Maximum Likelihood Estimation (MLE) and Expectation Maximization (EM) algorithm, which needs extensive computational expense.

Truncated distribution enables the travel time to be restricted within a certain limited range. Thus, excessively short or long travel times, which correspond to unreasonable values generated from the tail of standard distribution, can be excluded [4]. The upper and lower bounds can be defined by the maximum and minimum observed travel times, respectively, if the sample size is large enough [20]. As a result, the applicability of standard distributions can be improved if truncated distribution is adopted. However, the corresponding probability density function (PDF) of the truncated distribution should be modified in order to ensure that the integration of PDF in the specified range is equal to 1 , which increases the computation load to some extent.

2.2. Variability Patterns. Three main types of TTV can be generally found in the literature, that is, vehicle-to-vehicle variability that characterizes the difference between travel times of different vehicles traveling the similar route at the same time, period-to-period variability that relates to vehicles 
traveling the similar route at different periods within a day, and day-to-day variability that represents the travel time variations between similar trips at the same time period on different days $[16,23,24]$.

In order to identify the variability patterns of travel time, researchers have defined several reliability measures for quantitative analysis. TTR measures can be generally grouped into three categories, that is, the probability index, the statistical index, and the buffer time type index. The probability index mainly reflects the probability that the travel time can match the specified conditions in the form of probability distribution. The statistical index analyzes TTV based on historical data or real-time information, while the buffer time type index indicates the reservation time to ensure that the probability of reaching the destination is large enough from the travelers' point of view.

Moylan [25] and Rakha et al. [26] used standard deviation to measure the link TTV patterns. Yazici et al. [12] analyzed the differences in TTV patterns between urban roads and highways by calculating the coefficient of variation and found that higher travel times correspond to lower reliability on highways, yet correspond to higher reliability on urban roads. Kieu et al. [16] also employed the coefficient of variation to measure the variability of bus travel time on urban corridors. Van Lint and Van Zuylen [10] used width and skewness of the day-to-day TTD to predict freeway variability patterns. Pu [27] analytically examined a number of reliability measures and explored their mathematical relationships and interdependencies, including the 95th percentile travel time, standard deviation, coefficient of variation, travel time index, and planning time index. He concluded that the coefficient of variation is a good proxy for a range of reliability measures, and median-based buffer index or failure rate is recommended when TTDs are heavily skewed. Chase et al. [28] calculated thirteen reliability measures for 983 freeway segments from 15 -minute space mean speed data and explained the measures as to what they represent, which may help decision makers to effectively prioritize traffic management and geometric improvements. Moylan [25] considered the buffer time, planning time index, failure rate, and frequency of congestion of freeway segments. Alvarez and Hadi [29] measured the failure rate and misery index of general purpose lanes and high-occupancy toll lanes. The Florida Department of Transportation [30] used the percentage of travel time less than the median travel time plus a certain acceptable additional time such as the percentage of $5 \%, 10 \%, 15 \%$, and $20 \%$ above the expected travel time to estimate TTV. Table 1 presents the operational definitions of common reliability measures and the classes to which they belong.

To sum up, though TTD and variability patterns have been extensively investigated, limited studies shed light on the distinction at fine grained levels of spatial and temporal aggregation, for example, travel times for different road types during typical time periods. To fill this gap, this study conducts detailed analysis of travel time characteristics for each specific road type, for example, urban expressways, auxiliary roads of urban expressways, major roads, and secondary roads, based on a vast amount of proven vehicle data in the urban network of Beijing, China. Period-to-period and dayto-day variability are taken into comprehensive consideration. The results help reflect the travelers' preference to different road types and further enhance our understanding of traveling behavior in urban networks.

\section{Dataset}

In this study, the probe vehicle data collected in the urban network of Beijing, China, during one week from June 1st (Monday) to 7th (Sunday), 2015, were utilized. About every one minute, taxis equipped with GPS devices uploaded a set of instantaneous information, such as location, direction, speed, and being occupied or not occupied by passengers. Note that only the taxis occupied by passengers were considered, which are supposed to be closer to the behavior of regular vehicles. And enough data (at least five sets of traffic information) was uploaded within two minutes for each link. The data quality is good overall. The probe vehicle data were first preprocessed to remove erroneous information and a Kalman filtering process is utilized to achieve smoothing the vehicle trajectories. Then, by resorting to the map matching method, the travel speed data were further aggregated into every two minutes for each link within the network.

Note that in Beijing several two-way urban expressways, that is, Rings 2-5, enclose the urban area. The lengths of Rings $2-5$ are $33,48,65$ and $98 \mathrm{~km}$, respectively. The urban expressways are surrounded by auxiliary roads, and the auxiliary roads and urban expressways are connected by various types of interchanges with paired entrances and exits for merging or diverging traffic. Besides, major and secondary roads are widely distributed in the urban network.

In order to explore the TTD and variability patterns of urban roads, a large area inside the Third Ring Road in Beijing was selected and in total 200 links covering four road types, that is, urban expressways, auxiliary roads of urban expressways, major roads, and secondary roads, were exploited. Each type of road includes 50 links that are evenly distributed in the road network, and hence TTVs for each type of road are supposed to be represented comprehensively. Figure 1 shows the spatial distribution of different types of roads of interest.

\section{Day-of-Week Travel Time Distribution Analysis}

In this section, the probability distributions of travel time during different time periods were analyzed to investigate the characteristics of travel time variation.

4.1. Calculation Procedure of Travel Time Distribution Anal$y$ sis. As mentioned above, this study considered links from different road types. It is expected that various urban traffic states from different road types in different time periods can be described and compared in detail. For analyzing travel time distributions of each road type, we selected four typical periods of time, that is, peak hours on weekdays, off-peak hours on weekdays, peak hours on weekends, and off-peak hours on weekends. Peak hours represent the most congested 
TABLE 1: Travel time reliability measures.

\begin{tabular}{|c|c|c|}
\hline Category & Measure & Definition \\
\hline \multirow{3}{*}{ Probability index } & Variance & $\begin{array}{l}\text { The expected value of the square of the deviations of a random variable } \\
\text { from its mean value }\end{array}$ \\
\hline & Standard deviation & The square root of the variance \\
\hline & Coefficient of variation & Standard deviation divided by the average travel time \\
\hline \multirow{5}{*}{ Statistical index } & $K$ th percentile & $K$ th percentile of the travel time distribution \\
\hline & Skewness statistic & $\begin{array}{l}\text { The ratio of the difference between } 90 \text { th and } 50 \text { th percentile travel time to } \\
\text { the difference between } 50 \text { th and 10th percentile }\end{array}$ \\
\hline & Width statistic & $\begin{array}{l}\text { The ratio of the difference between } 90 \text { th and } 10 \text { th percentile travel time to } \\
\text { the 50th percentile travel time }\end{array}$ \\
\hline & Travel time index & The ratio of the average travel time to free-flow travel time \\
\hline & Planning time index & The ratio of 50th percentile travel time to free-flow travel time \\
\hline \multirow{4}{*}{$\begin{array}{l}\text { Buffer time type } \\
\text { index }\end{array}$} & Buffer time index & $\begin{array}{l}\text { The ratio of the difference between 95th percentile and the average travel } \\
\text { time to the average travel time }\end{array}$ \\
\hline & Failure/on-time performance & $\begin{array}{l}\text { Percent of trips with travel time less than, for example, } \\
1.1 * \text { median travel time, } 1.25 * \text { median travel time, and so on }\end{array}$ \\
\hline & Frequency of congestion & $\begin{array}{l}\text { Percent of time that the travel time is larger than double the free-flow travel } \\
\text { time }\end{array}$ \\
\hline & Misery index & $\begin{array}{l}\text { The average of the highest five percent of travel times divided by the } \\
\text { free-flow travel time }\end{array}$ \\
\hline
\end{tabular}

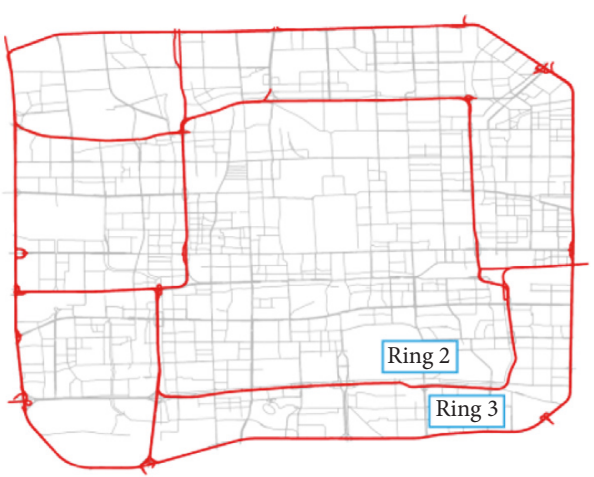

(a) Urban expressways

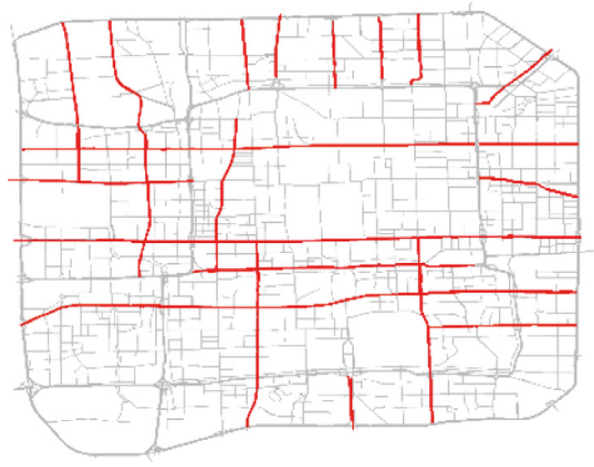

(c) Major roads

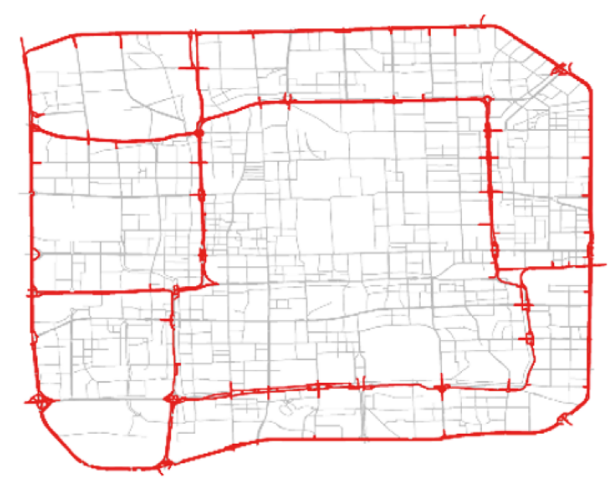

(b) Auxiliary roads of urban expressways

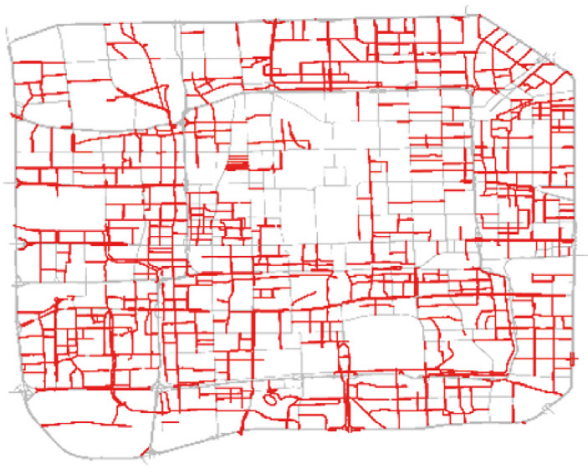

(d) Secondary roads

FIGURE 1: Spatial distribution of different types of roads. 
periods during the day while off-peak hours represent the smooth periods. They were distinguished for each road type by referring to the average travel time of the day. During each time period, it is assumed that link travel time is independent and identically distributed. For providing recommendations to large-scale network computing, such as path finding problems, four common types of single-mode probability distributions including normal, lognormal, gamma, and Weibull were employed to test whether they can fit the travel times desirably. The parameters of each distribution were estimated by the fitting functions in Matlab, such as normfit, lognfit, gamfit, and wblfit, using the travel time data of each period. The goodness-of-fit of each distribution was calculated using standard statistical tests such as Kolmogorov-Smirnov (K-S), Anderson-Darling (A-D), and chi-squared $\left(\chi^{2}\right)$ test at the 5\% significance level. If the $p$ value is larger than the significance level, the distribution is supposed to fit the original data significantly. The $\chi^{2}$ test is sensitive to the choice of the number of intervals. However, it seems that there is no optimal method to choose the width of the interval [4]. In this study, we set the initial number of intervals to be ten, which is also the default value set for $\chi^{2}$ test in Matlab. The complete calculation procedure is described below.

Step 1. Transform original vehicle speed data collected from probe vehicles into unit distance travel time. In this study, the unit distance was set as one hundred meters and the calculation of travel time was made in the unit of seconds per 100 meters. This step is to eliminate the influence of different road length on travel time analysis.

Step 2. Choose peak and off-peak hours according to the unit distance travel time on weekdays and weekends. For simplicity, one hour was set as the length of the analysis period.

Step 3. Determine parameter(s) of different distribution types from unit distance travel time. Here, the fitting functions in Matlab were used to estimate the optimal values of the parameter(s).

Step 4. Check the goodness-of-fit of four probability distributions by K-S test, A-D test, and $\chi^{2}$ test. Accordingly, whether each candidate distribution can be accepted by three statistical tests was examined.

Step 5. Calculate the average acceptance rates of each candidate distribution, and those for each time period and for each road type.

4.2. Analysis Results. Note that Step 4 in the above procedure aims to test whether the travel time data from each time period of each road type follow the four types of distributions using three statistical tests. Considering that 50 links are related for each road type, the unit distance travel times of one individual link in each period will be tested $(4 \times 3=) 12$ times. For a specific road type, candidate distribution, statistical test, and time period, the tests will be performed $(50 \times 5=) 250$ times for weekdays and $(50 \times 2=) 100$ times for weekends. The goodness-of-fit results of TTD for different road types during different periods are presented in Table 2.

The following presents the analysis of distribution fitting results investigated from three perspectives, that is, probability distribution types, time periods, and road types.

4.2.1. Distribution Types. First, the best-fitted distribution type was analyzed on the whole for altogether 16 time periods through comparing the average acceptance rates of each distribution. It was found that lognormal distribution has the highest average acceptance rates; that is, $14 / 16=87.5 \%$. It is worth noting that the best-fitted distribution type for TTD of urban expressways during peak hours on weekends is Weibull distribution and for TTD of the auxiliary roads of urban expressways during off-peak hours on weekends is gamma distribution. However, even in such cases the difference between the acceptance rates of lognormal distribution and the best-fitted distribution is minimal and less than $1 \%$. Then, the best-fitted distribution type was analyzed within each time period. The results showed that the average acceptance rates of lognormal, gamma, normal, and Weibull distribution are $83.7 \%, 81.9 \%, 78.8 \%$, and $78.2 \%$, respectively. Apparently, lognormal distribution seems superior compared with other three types of distribution.

4.2.2. Time Periods. In this study, the best-fitted time period was defined as the one whose average acceptance rate of all 12 statistical tests, that is, K-S, A-D, and $\chi^{2}$ tests, is highest. Accordingly, three best-fitted time periods were identified as off-peak hours of major roads on weekdays, off-peak hours of major roads on weekends, and off-peak hours of auxiliary roads of urban expressways on weekends, with the average acceptance rates of $93.0 \%, 92.8 \%$, and $92.7 \%$, respectively. By comparison, three worst-fitted time periods are peak hours of secondary roads on weekends, peak hours of secondary roads on weekdays, and peak hours of urban expressways on weekdays, with the average acceptance rates of $68.4 \%, 67.7 \%$, and $67.1 \%$, respectively. It is interesting to notice that the average acceptance rates on weekdays were always less than on weekends for each road type, and those during peak hours were always less than during off-peak hours within each time period except for the weekends of urban expressway. It helps demonstrate that the typically different traffic states during peak and off-peak hours on weekdays and weekends make a difference of travel time characteristics.

4.2.3. Road Types. In practice, travel times of different road types are supposed to have distinct characteristics. When mixing weekdays and weekends for each road type, the average acceptance rates of urban expressways, auxiliary roads of urban expressways, major roads, and secondary roads are $73.6 \%, 89.0 \%, 89.6 \%$, and $70.4 \%$, respectively. It implies that the travel times on auxiliary roads of urban expressways and major roads fitted standard distributions better than the other types of road. The related TTV patterns of each road type will be analyzed in terms of reliability measures in the following. 


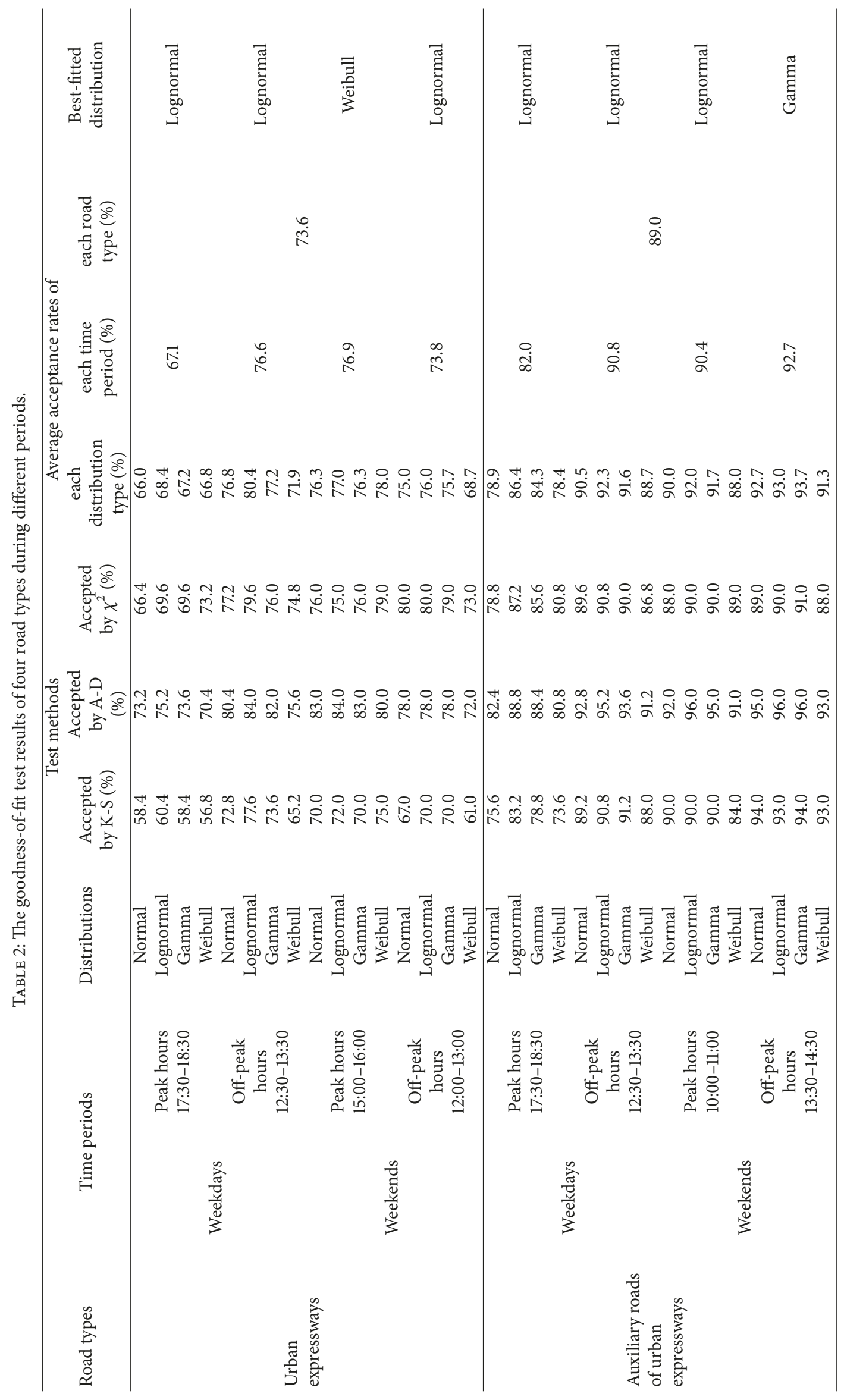




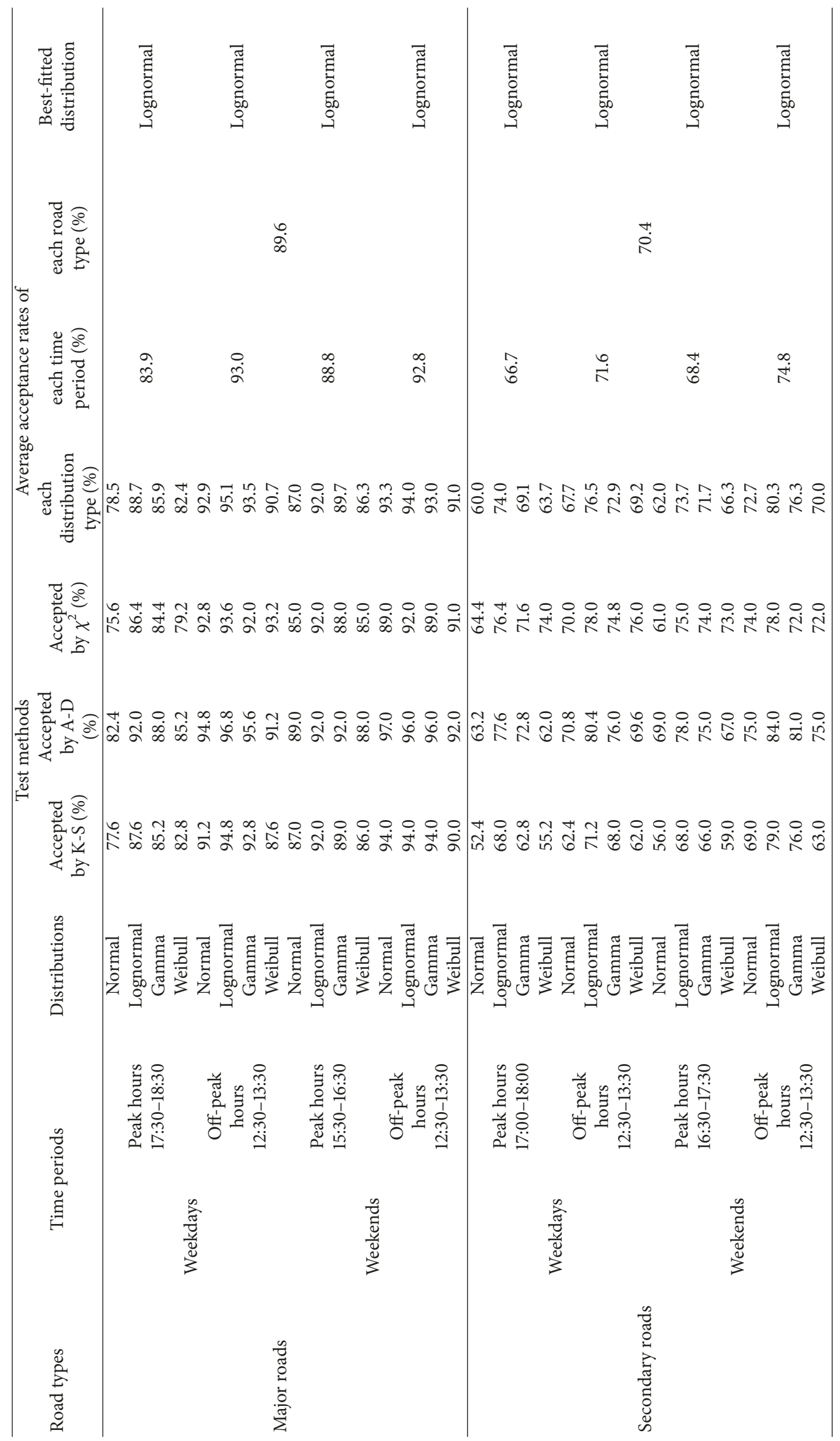




\section{Day-of-Week Variability Patterns of Different Road Types}

The temporal variation of travel times for each road type was analyzed by exploring the variability patterns, which is beneficial for traffic management agencies to make sophisticated strategies and for travelers to make smart route choices. To this end, four reliability measures were employed, that is, unit distance travel time, coefficient of variation, buffer time index, and punctuality rate. The detailed analysis results are provided below.

5.1. Unit Distance Travel Time. As mentioned before, we transformed the original travel time data from probe vehicles into unit distance travel time. According to the state-of-theart studies [1], travel times at different quantiles have been commonly used to present the variation tendency changes through the whole day. Thus, we selected the 15th percentile, average, and the 95th percentile travel time to study the variability patterns. The 15 th percentile travel time was usually regarded as free flow travel time, and the 95th percentile travel time as the time budget within which travelers will arrive on time. The average travel time of each road type can be calculated as $\overline{\mathrm{TT}_{X, w}}$ in

$$
\overline{\mathrm{TT}_{X, w}}=\frac{\sum_{n=1}^{N} \mathrm{TT}_{X, n, w}}{N \times m},
$$

where $\overline{\mathrm{TT}_{X, w}}$ is the average value of all links travel time for road type $X$ within time window $w$ during $N$ days; $\mathrm{TT}_{X, n, w}$ is link travel time for road type $X$ within time window $w$ on day $n ; m$ is the number of links, that is, 50 in this study.

Figure 2 illustrates the variability of different percentile unit distance travel times for each road type through the day. The main findings are summarized below.

On weekdays, the morning and afternoon peaks, which look like two bumps (camel shape) and highly skewed distributions, can be obviously distinguished for each road type. Note that the duration of peak hours on urban expressways is comparatively longer than the other types of road, indicating that urban expressways bear higher traffic demand in accordance with its designed function. Moreover, the TTDs over the four hierarchical road types seem to follow a similar and predictable pattern over the day. On the other hand, on weekends all types of roads did not have obvious morning and evening peaks as on weekdays and the unit distance travel times were shortened on the whole to a large extent. It is also interesting to notice that secondary roads tend to have larger unit distance travel times in daytime on both weekdays and weekends. These events may be due to the limited traffic capacity of secondary roads, where it is easier to reach saturation than other road types.

More detailed analysis shows that the morning peak on weekdays appeared around 8:00-9:00 and afternoon peak around 18:00-19:00, which well agrees with the commuting time periods. On weekends, the peak hours through the day appeared around 11:00-12:00 or 16:00-17:00, indicating distinctly different traveling behavior and variability patterns compared with weekdays. Furthermore, during the night periods, for example, 0:00-6:00 and 20:00-0:00, the 15th percentile, average, and the 95th percentile travel times of all road types were short and close to each other. In particular, the 15th percentile travel times of all road types were almost unchanging through the day on both weekdays and weekends. While, during the daytime, for example, 6:00-10:00, the average and the 95th percentile travel times increased significantly. Especially during peak hours, the 95th percentile travel time was about two to three times of the average travel time on weekdays and weekends and as much as six to nine times of the 15 th percentile travel time on weekdays and three to five times on weekends.

5.2. Coefficient of Variation. Although the standard deviation is one of the most commonly used variability measures, the absolute value of the standard deviation may yield questionable inferences about the variability [11]. Thus, variability patterns were measured using the coefficient of variation (CV) which normalizes the standard deviation based on the average travel time of the selected time periods. The CV of travel times for road type $X$ within time window $w$ during $N$ days can be calculated as $\mathrm{CV}_{X, w}$ in

$$
\mathrm{CV}_{X, w}=\frac{(1 /(N \times m)) \sum_{n=1}^{N}\left(\mathrm{TT}_{X, n, w}-\overline{\mathrm{TT}_{X, w}}\right)^{2}}{\overline{\mathrm{TT}_{X, w}}}
$$

The CVs of travel times for each road type are shown in Figure 3. Overall, it is interesting to notice that though weekdays and weekends have distinctly different traffic characteristics, the CVs of travel times for each road type did not have such significant difference. It implies that TTR measures can help interpret the essential characteristics of variability patterns.

On both weekdays and weekends, the CVs for urban expressways in daytime were relatively smaller and had a narrower fluctuation range compared with major roads and secondary roads. It indicates relatively stable traffic states of uninterrupted flow and reliable travel times on urban expressways. It should be pointed out that, during the periods of 1:00-3:00, the CVs for urban expressway fluctuated significantly. One possible reason is that during such periods the vehicle speed data collected from probe vehicles were scarce and related to greater randomness of driving behavior.

Furthermore, on weekdays the maximum CVs of urban expressways and auxiliary roads of urban expressways through the day were around 1 . The secondary roads had a large value of the maximum $\mathrm{CV}$ around 1.6 and the major roads around 1.8. On weekends, the maximum $\mathrm{CV}$ of urban expressways through the day was about 1 . The values for auxiliary roads of urban expressways and secondary roads were around 1.4, and major roads still had the largest maximum CV of around 1.8. The interrupted nature of travel flows on major roads makes travel times more variable. Due to interactions between volatile traffic regimes and signal control strategies, the resulting travel times on major roads often present various distributions under different levels of congestion [6] and thus larger CVs in operation. 


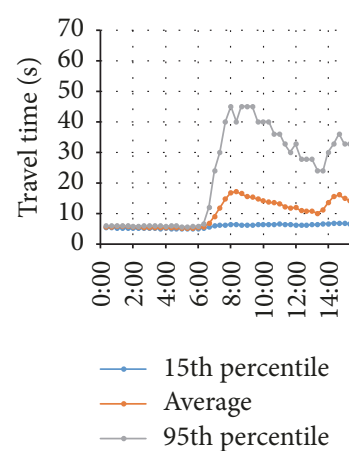

(a) Urban expressways on weekdays

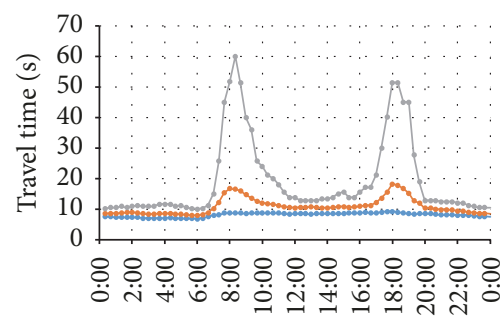

- 15th percentile

$\therefore$ Average

$\because$ 95th percentile

(c) Auxiliary roads of urban expressways on weekdays

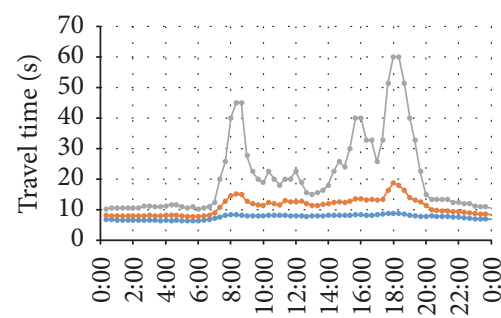

-15 th percentile

$\longrightarrow$ Average

- 95th percentile

(e) Major roads on weekdays

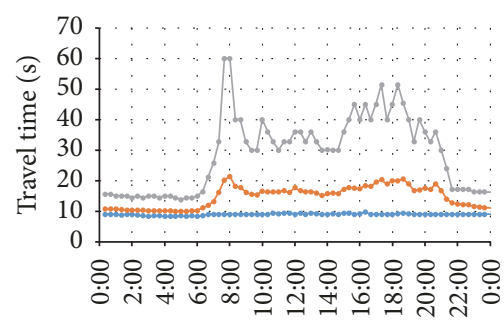

$\longrightarrow$ 15th percentile

- Average

- 95 th percentile

(g) Secondary roads on weekdays

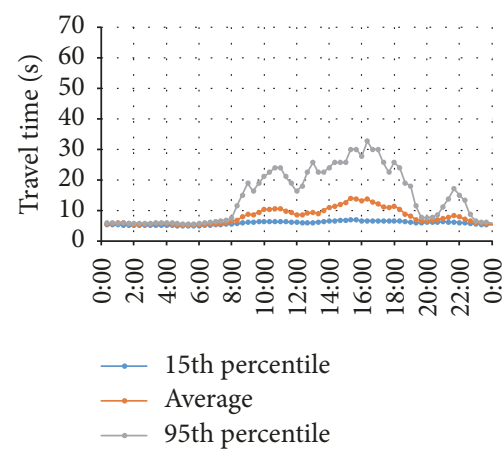

(b) Urban expressways on weekends

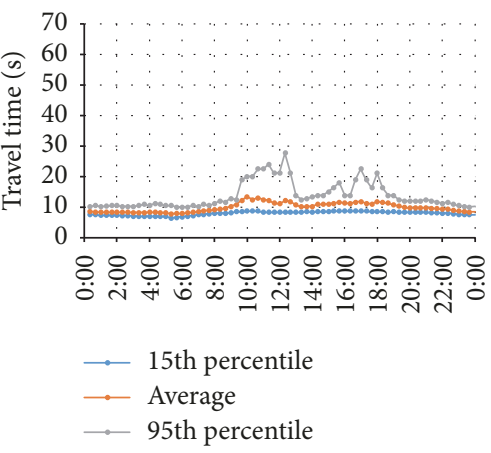

(d) Auxiliary roads of urban expressways on weekends

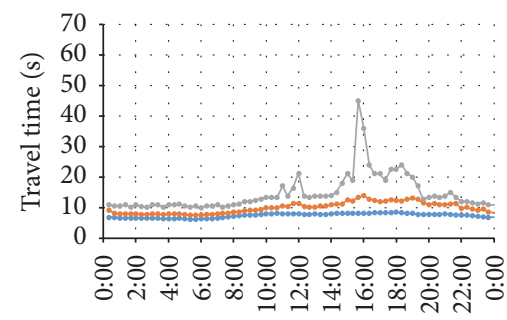

$\because$ 15th percentile

$\longrightarrow$ Average

- 95th percentile

(f) Major roads on weekends

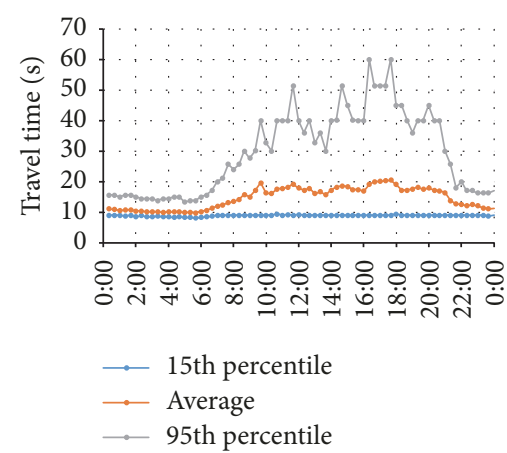

(h) Secondary roads on weekends

FIgURE 2: Unit distance travel time for different types of road through the day. 


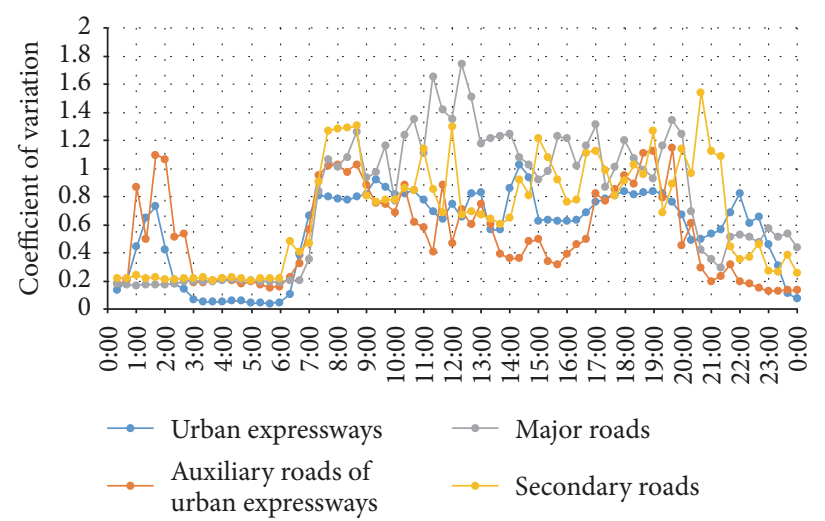

(a) On weekdays

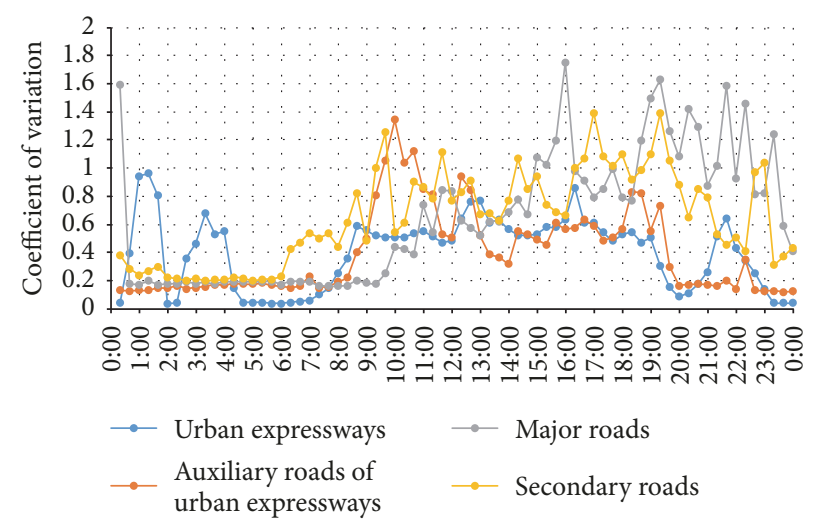

(b) On weekends

FIgURE 3: Coefficient of variation through the day.

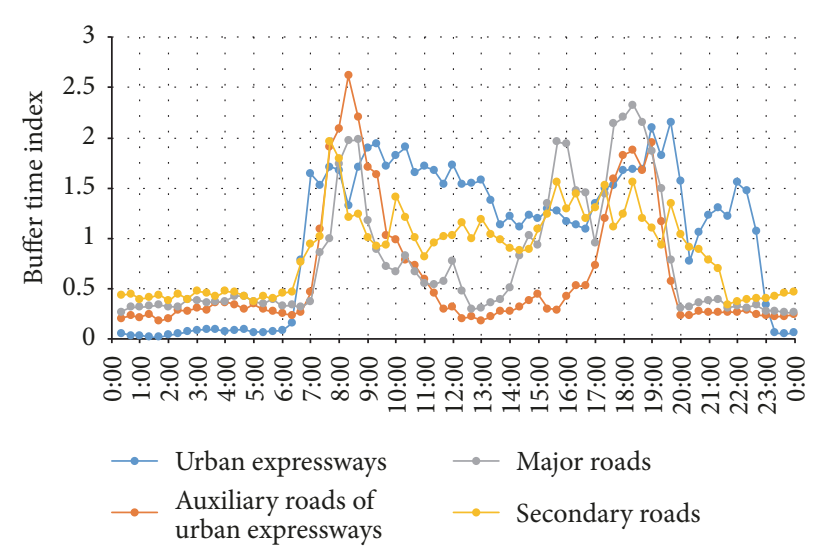

(a) On weekdays

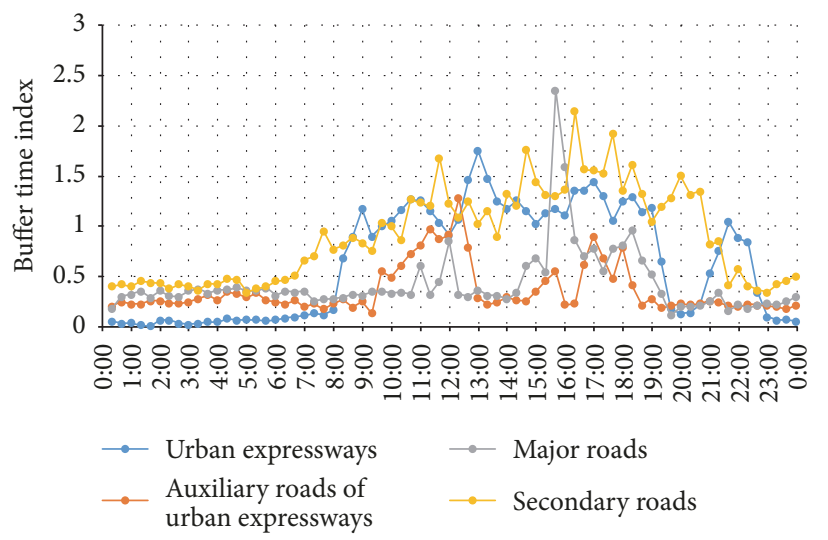

(b) On weekends

FIgURE 4: Buffer time index through the day.

5.3. Buffer Time Index. The buffer time index (BTI) represents the additional time that travelers have to spend on the basic of average travel time when they would like to arrive at their destination on time [31]. The BTI for road type $X$ can be expressed as $\mathrm{BTI}_{X}$ in

$$
\mathrm{BTI}_{X}=\frac{\mathrm{TT}_{X, w, 95 \mathrm{th}}-\overline{\mathrm{TT}_{X, w}}}{\overline{\mathrm{TT}_{X, w}}},
$$

where $\mathrm{TT}_{X, w, 95 \text { th }}$ is the 95th percentile travel time for road type $X$ within time window $w$ during $N$ days.

The BTIs of travel times for each road type are shown in Figure 4. It was found that the BTIs seem to have shapes very close to the 95th percentile travel times in Figure 2. In more detail, on weekdays BTIs had obvious peak values in the morning and afternoon, for example, around 8:00-9:00 and 18:00-20:00, for auxiliary roads of urban expressways and major roads. The BTIs for urban expressways were minimal until 6:00 and then maintained larger values ranging from 1 to 2 , implying volatile and unreliable traffic states through the day. Though, with relatively lower values, the BTIs for secondary roads shared the similar changing tendency as urban expressways. Note that the maximum value of BTIs among four road types on both weekdays and weekends was more than 2, which implies that the drivers would spend three times as much as the average travel time in that period when planning trips to guarantee they would have 95 percent of the possibilities to arrive on time. The minimum value of BTI was close to 0 and related to urban expressways. For auxiliary roads of urban expressways and major roads the minimum value of BTI was around 0.25 , while it was about 0.5 for secondary roads, implying usually unreliable travel times provided by secondary roads.

$\mathrm{Pu}$ [27] pointed out that some reliability metrics may be inconsistent in their depictions of reliability, such as the case of BTI that may remain constant for different values of CV. The same conclusions can be drawn in this study. It was obvious that on weekdays the CVs of urban expressways and auxiliary roads of urban expressways had a large fluctuation in early morning, for example, around 0:00-3:00; however, the BTIs of the same road types were stable during that period. Similar phenomena can also be recognized on weekends, for instance, for urban expressways in early morning, for example, around 0:00-4:00, as well as major roads 


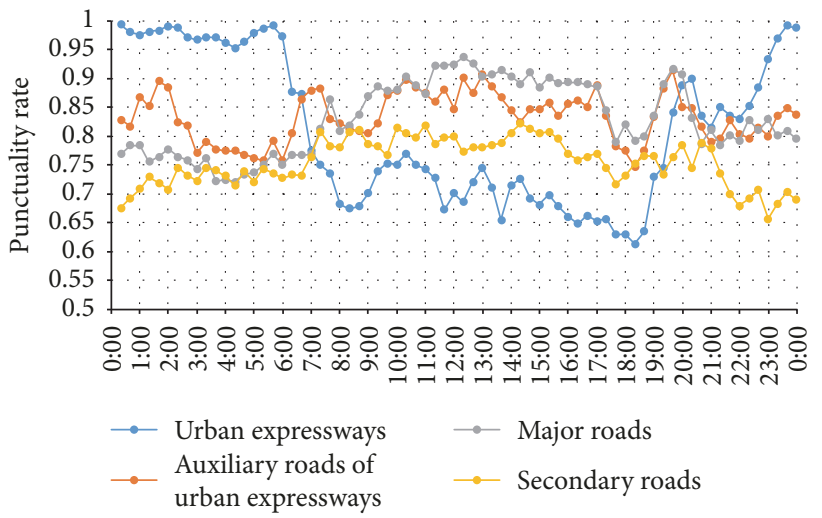

(a) On weekdays

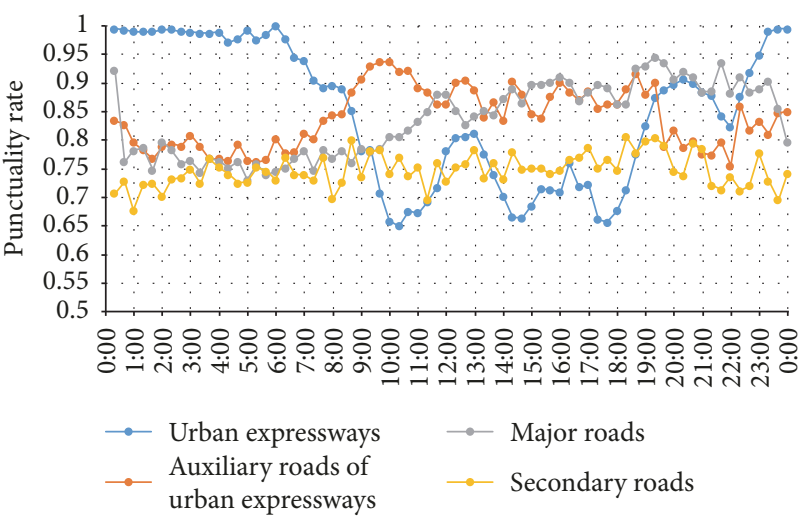

(b) On weekends

FIGURE 5: Punctuality rates through the day.

in the late evening, for example, around 20:00-0:00. Hence, we may need to consider the changes of multiple indicators comprehensively to accurately measure the road performance.

5.4. Punctuality Rate. In this study, the punctuality rate (PR) was defined as the probability that the travel times were less than or equal to 1.1 times as much as the average travel time of road type $X$ within time window $w$ during $N$ days. To a certain extent, PR can reflect the operational performance of different road types. The higher the $\mathrm{PR}$ is, the more desirable the road performance is. The reason we prefer average travel time to median travel time when calculating PR is that the skew statistics of most links during most time periods are larger than 1 indicating that the median travel time is smaller than average travel time. As a result, median-based PR may underestimate the operational performance. The PR can be expressed as

$$
\mathrm{PR}_{X}=\sum_{n=1}^{N} P\left\{\mathrm{TT}_{X, n, w} \leq 1.1 \times \overline{\mathrm{TT}_{X, w}}\right\},
$$

where $\mathrm{PR}_{X}$ is the punctuality rate for road type $X$ within time window $w$ during $N$ days.

The PRs of travel times for each road type are shown in Figure 5. It is noteworthy that from late evening till early evening, for example, 22:00-7:00, on both weekdays and weekends, the PRs of urban expressways were over 0.9 and remained the highest among four types of roads. During the same periods, the PRs of auxiliary roads of urban expressways and major roads fluctuated between 0.7 and 0.9 , also indicating desirable operational performance with less traffic demand. By comparison, secondary roads had relatively lower PRs, that is, less than 0.75 .

It is worth noting that during the daytime after 7:00 the PRs of urban expressways began to decline rapidly and remained the lowest among four road types. On the other hand, the PRs of auxiliary roads of urban expressways and major roads began to rise slightly and remained the top two among four road types. For secondary roads, the PRs remained relatively stable through the day, and even higher than urban expressway during the daytime period of 7:00-19:00 on weekdays and 9:00-19:00 on weekends. It implies that, with the increasing commuting traffic demand, most of travelers might prefer to choose urban expressways first and they increased the possibility of traffic congestion on urban expressways. Meanwhile, the PRs of auxiliary roads of urban expressways and major roads became higher. Furthermore, it is interesting to notice that auxiliary roads of urban expressways and major roads have similar changing tendency of PRs as well as unit distance travel time and BTI and thus had similar travel time variation patterns.

\section{Summary and Conclusions}

In order to investigate the volatile characteristics of travel time in an urban network, this study explored the urban TTD and the variability patterns of different road types by using the probe vehicle data collected inside the Third Ring Road in Beijing. Different from the previous studies, in total, 200 links covering four road types, that is, urban expressways, auxiliary roads of urban expressways, major roads, and secondary roads, were exploited to provide more detailed analysis of travel time characteristics for each specific road type. The major achievements are summarized below, which may be beneficial to travelers for making reliable route choices and to traffic engineers for deploying sophisticated management and control.

(1) Link TTDs are characterized using probe vehicle data. Four common probability distributions, including normal, lognormal, gamma, and Weibull, are subjected to standard statistical tests such as K-S, $\mathrm{A}-\mathrm{D}$, and chi-squared test. The periods of interest were divided into four groups, that is, peak hours on weekdays, off-peak hours on weekdays, peak hours on weekends, and off-peak hours on weekends.

(2) Lognormal distribution is superior compared with other three types of distribution. The average acceptance rates on weekdays were always less than on weekends for each road type, and those during peak 
hours were always less than during off-peak hours within each time period except for the weekends of urban expressway. The travel times on auxiliary roads of urban expressways and major roads fitted standard distributions better than the other types of road. It can be implied that travel time characteristics vary from different traffic states and road types. Moreover, fitting results of link TTDs can provide guidance for determining path TTDs and network modeling.

(3) Four reliability measures, that is, unit distance travel time, CV, BTI, and PR, were used to quantify the day-of-week TTV patterns of different road types. In general, various indicators can reflect the change trend of traffic states collaboratively. However, some reliability measures may be inconsistent in their depictions of TTV, such as the case of BTI that may remain constant for different values of $\mathrm{CV}$. As a result, it is more reasonable and accurate to consider the changes of multiple indicators comprehensively when measuring the road performance.

(4) On weekdays, the morning and afternoon peaks could be distinguished easily for urban expressways, auxiliary roads of urban expressways, and major roads, all of which appeared around 8:00-9:00 and 18:00-19:00, respectively. However, on weekends, all types of roads did not have obvious morning and afternoon peaks, and the peak through the day appeared around 11:00-12:00 or 16:00-17:00, indicating distinctly different traveling behavior and variability patterns compared with weekdays. Moreover, travelers can attempt to avoid traveling during peak hours to save time, and traffic management departments may target peak hours for improving traffic conditions.

(5) Auxiliary roads of urban expressways and major roads had similar changing tendency of $P R$ as well as unit distance travel time and BTI, thus sharing similar TTV patterns. The travel times of auxiliary roads of urban expressways and major roads appeared more stable and reliable than other road types in daylight, and urban expressways had the most reliable travel times at night. It implies that with the increasing commuting traffic demand, most of travelers might prefer to choose urban expressways first and increase the possibility of traffic congestion on urban expressways.

In the future work, to enhance the effectiveness and reliability of the results, it is expected to adopt larger scale probe data in the time-space dimension or to integrate the data from different sources, for example, fusing probe data with other data collected by traffic sensors such as loop detectors and camera [32]. Furthermore, since the probability distribution types in this study only include four standard distributions, examining the goodness-of-fit of other distributions, for example, multimode distribution and truncated distribution, will also be conducted. On the other hand, additional reliability measures are supposed to be incorporated for more comprehensive evaluation. Last but not least, travel time forecast problem can also be delved into $[33,34]$.

\section{Conflicts of Interest}

The authors declare that they have no conflicts of interest.

\section{Acknowledgments}

The authors acknowledge the National Nature Science Foundation of China (U1564212 and 51508014) and the Fundamental Research Funds for the Central Universities for kind support of this research.

\section{References}

[1] SHRP2, Second Strategic Highway Research Program, 2010, http:// www.trb.org/StrategicHighwayResearchProgram2SHRP2/Blank2 .aspx.

[2] P. Chen, G. Yu, X. Wu, Y. Ren, and Y. Li, "Estimation of redlight running frequency using high-resolution traffic and signal data," Accident Analysis \& Prevention, vol. 102, pp. 235-247, 2017.

[3] M. Martchouk, F. Mannering, and D. Bullock, "Analysis of Freeway Travel Time Variability Using Bluetooth Detection," Journal of Transportation Engineering, vol. 137, no. 10, pp. 697704, 2011.

[4] W. Zeng, T. Miwa, Y. Wakita, and T. Morikawa, "Application of Lagrangian relaxation approach to $\alpha$-reliable path finding in stochastic networks with correlated link travel times," Transportation Research Part C: Emerging Technologies, vol. 56, pp. 309-334, 2015.

[5] X. Wang, H. Liu, R. Yu, B. Deng, X. Chen, and B. Wu, "Exploring operating speeds on urban arterials using floating car data: Case study in Shanghai," Journal of Transportation Engineering, vol. 140, no. 9, Article ID 04014044, 2014.

[6] P. Chen, K. Yin, and J. Sun, "Application of finite mixture of regression model with varying mixing probabilities to estimation of urban arterial travel times," Transportation Research Record, vol. 2442, pp. 96-105, 2014.

[7] K. Lyman and R. L. Bertini, "Using travel time reliability measures to improve regional transportation planning and operations," Transportation Research Record, no. 2046, pp. 1-10, 2008.

[8] P. Chen, C. Ding, G. Lu, and Y. Wang, "Short-term traffic states forecasting considering spatial-temporal impact on an urban expressway," Transportation Research Record, vol. 2594, pp. 6172, 2016.

[9] F. Lei, Y. Wang, G. Lu, and J. Sun, "A travel time reliability model of urban expressways with varying levels of service," Transportation Research Part C: Emerging Technologies, vol. 48, pp. 453-467, 2014.

[10] J. W. C. Van Lint and H. J. Van Zuylen, "Monitoring and predicting travel time reliability: using width and skew of dayto-day travel time distribution," Transportation Research Record, vol. 1917, pp. 54-62, 2005.

[11] M. Yazici, C. Kamga, and K. Mouskos, "Analysis of travel time reliability in New York City based on day-of-week and time-ofday periods," Transportation Research Record, no. 2308, pp. 8395, 2012.

[12] M. A. Yazici, C. Kamga, and K. Ozbay, "Highway versus urban roads: Analysis of travel time and variability patterns based on facility type," Transportation Research Record, vol. 2442, pp. 5361, 2014. 
[13] M. A. P. Taylor, "Travel time variability - the case of two public modes," Transportation Science, vol. 16, no. 4, pp. 507-521, 1982.

[14] E. Mazloumi, G. Currie, and G. Rose, "Using GPS data to gain insight into public transport travel time variability," Journal of Transportation Engineering, vol. 136, no. 7, Article ID 006007QTE, pp. 623-631, 2010.

[15] E. B. Emam and H. Al-Deek, "Using real-life dual-loop detector data to develop new methodology for estimating freeway travel time reliability," Transportation Research Record, no. 1959, pp. 140-150, 2006.

[16] L.-M. Kieu, A. Bhaskar, and E. Chung, "Public transport travel-time variability definitions and monitoring," Journal of Transportation Engineering, vol. 141, no. 1, Article ID 04014068, 2015.

[17] Y. Ji and H. Zhang, "Travel Time Distributions on Urban Streets: Estimation with Hierarchical Bayesian Mixture Model and Application to Traffic Analysis with High-Resolution Bus Probe Data," in Transportation Research Board 92nd Annual Meeting, 2013.

[18] F. Guo, Q. Li, and H. Rakha, "Multistate travel time reliability models with skewed component distributions," Transportation Research Record, vol. 2315, pp. 47-53, 2012.

[19] F. Guo, H. Rakha, and S. Park, "Multistate model for travel time reliability," Transportation Research Record, no. 2188, pp. 46-54, 2010.

[20] P. Cao, T. Miwa, and T. Morikawa, "Modeling Distribution of Travel Time in Signalized Road Section Using Truncated Distribution," Procedia - Social and Behavioral Sciences, vol. 138, pp. 137-147, 2014.

[21] Y. Wang, W. Dong, L. Zhang et al., "Speed modeling and travel time estimation based on truncated normal and lognormal distributions," Transportation Research Record, no. 2315, pp. 6672, 2012.

[22] Y. Nie, X. Wu, J. F. Dillenburg, and P. C. Nelson, "Reliable route guidance: A case study from Chicago," Transportation Research Part A: Policy and Practice, vol. 46, no. 2, pp. 403-419, 2012.

[23] M. Yildirimoglu, Y. Limniati, and N. Geroliminis, "Investigating empirical implications of hysteresis in day-to-day travel time variability," Transportation Research Part C: Emerging Technologies, vol. 55, pp. 340-350, 2015.

[24] R. B. Noland and J. W. Polak, "Travel time variability: a review of theoretical and empirical issues," Transport Reviews, vol. 22, no. 1, pp. 39-54, 2002.

[25] E. Moylan, "Performance of Reliability Metrics on Empirical Travel Time Distributions," in Transportation Research Board 93rd Annual Meeting, 2014.

[26] H. Rakha, I. El-Shawarby, M. Arafeh, and F. Dion, "Estimating path travel-time reliability," in Proceedings of the ITSC 2006: 2006 IEEE Intelligent Transportation Systems Conference, pp. 236-241, September 2006.

[27] W. Pu, "Analytic relationships between travel time reliability measures," Transportation Research Record, vol. 2254, pp. 122130, 2011.

[28] R. Chase, B. Williams, and N. Rouphail, "Detailed Analysis of Travel Time Reliability Performance Measures from Empirical Data," in Transportation Research Board 92nd Annual Meeting, 2013.

[29] P. Alvarez and M. Hadi, “Time-variant travel time distributions and reliability metrics and their utility in reliability assessments," Transportation Research Record, no. 2315, pp. 81-88, 2012.
[30] Florida Department of Transportation, The Florida Reliability Method. In Florida's Mobility Performance Measures Program, 2000.

[31] Federal Highway Administration (FHWA), Travel Time Reliability: Making It There On Time, All The Time, 2006.

[32] Y. Wiseman, "Real-time monitoring of traffic congestions," in Proceedings of the 2017 IEEE International Conference on Electro Information Technology, EIT 2017, pp. 501-505, USA, May 2017.

[33] Z. Zhang, Y. Wang, P. Chen, Z. He, and G. Yu, "Probe data-driven travel time forecasting for urban expressways by matching similar spatiotemporal traffic patterns," Transportation Research Part C: Emerging Technologies, vol. 85, pp. 476493, 2017.

[34] J. J. Tang, F. Liu, Y. J. Zou, W. B. Zhang, and Y. H. Wang, "An improved fuzzy neural network for traffic speed prediction considering periodic characteristic," IEEE Transactions on Intelligent Transportation Systems, vol. 18, pp. 2340-2350, 2017. 


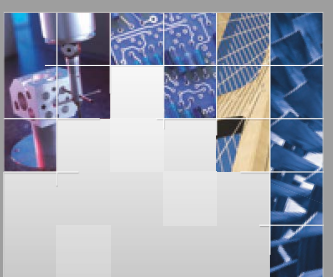

\section{Enfincering}
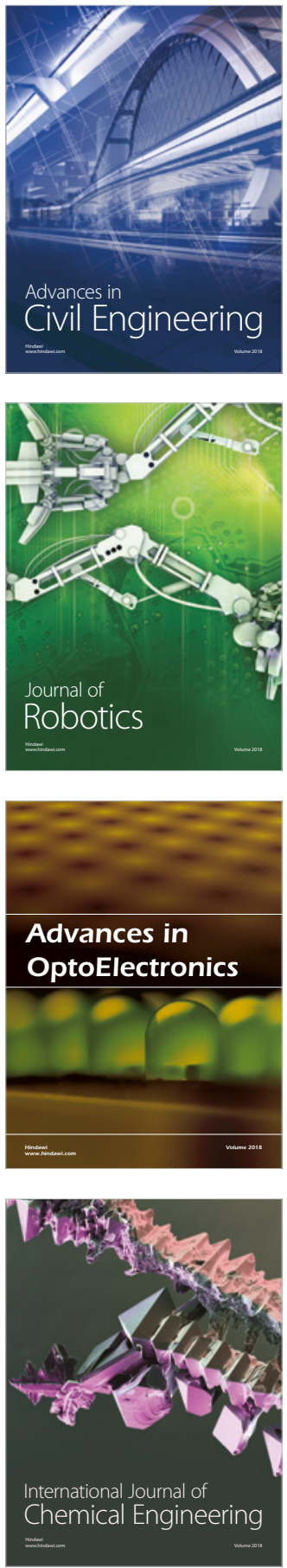

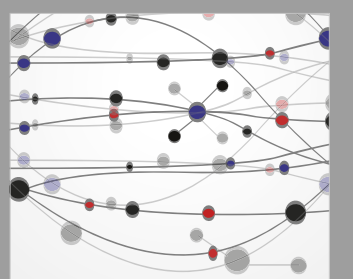

\section{Rotating \\ Machinery}

The Scientific World Journal

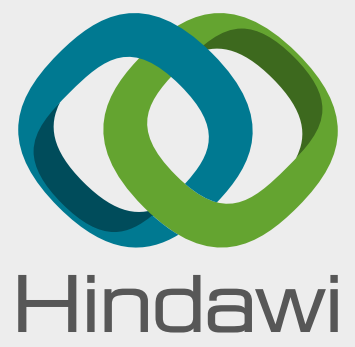

Submit your manuscripts at

www.hindawi.com
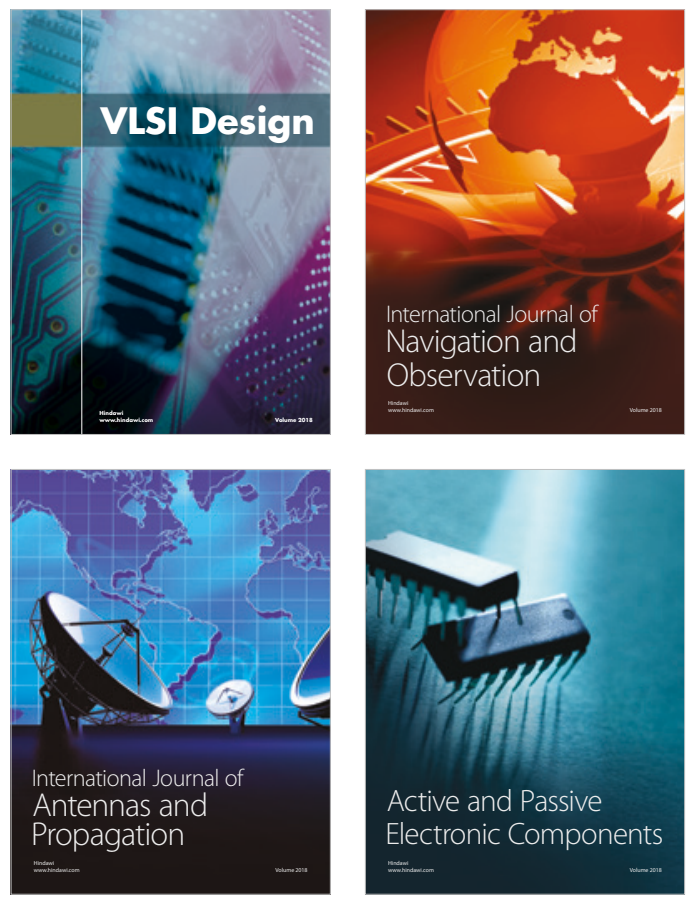
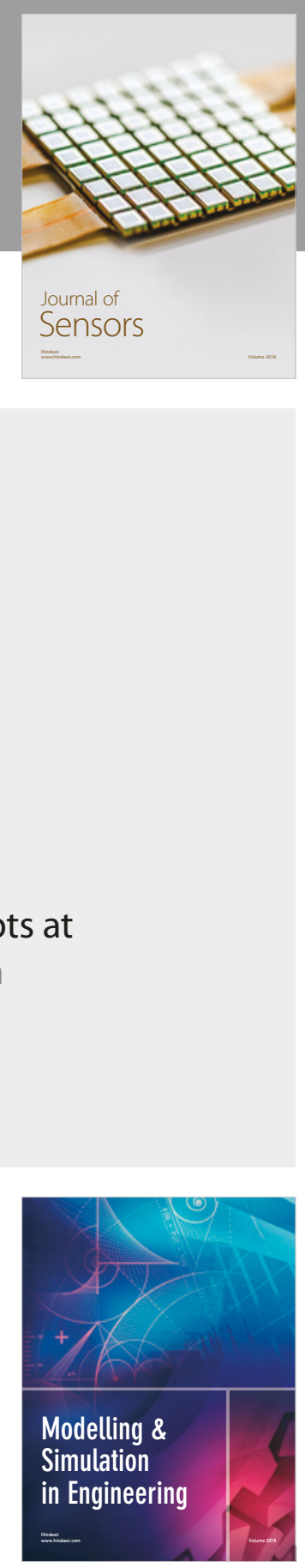

\section{Advances \\ Multimedia}
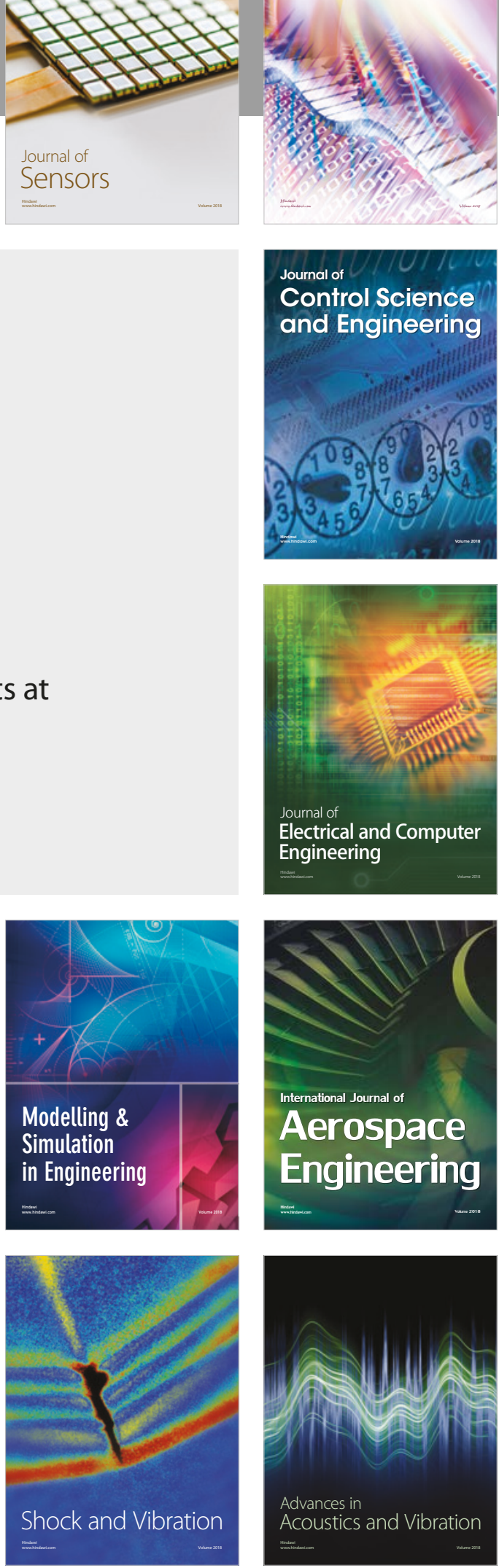